\title{
Hemorragia digestiva masiva como manifestación de tumor mucinoso apendicular: Reporte de caso
}

\author{
Massive digestive hemorrhage as a manifestation of an \\ appendicular mucinous tumor: Case report
Natalia Rodríguez Rosales, ${ }^{*}$ Hernando Kuan Casas, ${ }^{*}$ Douglas Ortiz Espinel, ${ }^{\ddagger}$ Lucas Gómez Ibáñez, ${ }^{\star}$ Javier Andrés Morales Morales, ${ }^{*}$ Álvaro Andrés González Alférez ${ }^{*}$
* Hospital Universitario Clínica San Rafael.
₹ Unidad de Gastroenterología, Hospital Universitario Clínica San Rafael. \\ Universidad Militar Nueva Granada, Bogotá, D.C., Colombia.
}

\section{RESUMEN}

Se reporta un caso en Bogotá, Colombia de hombre de 63 años con dolor abdominal de un mes de evolución, asociado a deposiciones diarreicomelénicas, que asiste a la institución de tercer nivel con tendencia a la hipotensión. Se realiza endoscopía de vías digestivas (EVDA) sin evidencia de lesiones o sangrado. Colonoscopía con mala preparación, con restos hemáticos a nivel de válvula ileocecal sin evidenciar sitio de sangrado. Se realiza nuevamente EVDA y colonoscopía sin evidencia de sangrado activo, con restos hemáticos a nivel de válvula ileocecal. Se realiza gammagrafía con leucocitos marcados reportando hemorragia digestiva baja en segmento proximal de colon ascendente. El paciente persiste con evacuaciones melénicas, requerimiento de soporte vasopresor, trasfusión de hemoderivados y dolor abdominal, por lo que se realiza laparotomía con enteroscopía intraoperatoria encontrando orificio apendicular con abundante sangrado en ciego y colon ascendente y algunas lesiones angiectásicas a nivel de íleon distal. Se decide realización de hemicolectomía derecha con resección de íleon terminal con anastomosis ileotransversa. Presentó adecuada evolución clínica. Reporte de patología confirma neoplasia mucinosa apendicular de $1 \mathrm{~cm}$, de bajo grado, no infiltrante, sin evidencia de lesiones isquémicas y 11 ganglios linfáticos del tejido graso sin compromiso tumoral u otras lesiones.

Palabras clave: Tumor mucinoso apendicular, sangrado digestivo bajo masivo.
ABSTRACT

We report a case of a 63-year-old male patient in Bogota, Colombia with a one month history of abdominal pain, associated with diarrheal stools, bloody diarrheal, that was admitted to our third level institution with a tendency to hypotension. Endoscopy was performed without lesions or bleeding. Colonoscopy with poor preparation found residual blood in the ileocecal valve without showing a bleeding site. Endoscopy and colonoscopy were performed again, without evidence of active bleeding, with blood remains. A labeled white blood cells scintigraphy reporting low digestive hemorrhage in the proximal segment of the ascending colon. The patient persisted with bloody stools, vasopressor support, transfusion and abdominal pain. He underwent to laparotomy with intraoperative enteroscopy finding appendicular orifice with large bleeding in the cecum and ascending colon and some angiectatic lesions at the distal ileum. It was decided to perform a right hemicolectomy with resection of the terminal ileum with ileotransverse anastomosis, with adequate clinical follow up. Pathological examination confirmed low-grade appendicular mucinous neoplasia, $1 \mathrm{~cm}$ in diameter, without infiltration, without evidence of ischemic lesions, 11 lymph nodes of fatty tissue without tumor involvement, without other lesions.

Keywords: Appendiceal mucinous tumor, lower gastrointestinal bleeding.

\section{Recibido: 26/07/2020. Aceptado: 05/08/2020. \\ Correspondencia: Natalia Rodríguez Rosales \\ E-mail: natarods88@gmail.com}

Citar como: Rodríguez RN, Kuan CH, Ortiz ED, Gómez IL, Morales MJA, González AÁA. Hemorragia digestiva masiva como manifestación de tumor mucinoso apendicular: Reporte de caso. Rev Mex Cir Endoscop. 2020; 21 (4): 206-209. https://dx.doi.org/10.35366/101220 


\section{INTRODUCCIÓN}

La hemorragia de vías digestivas bajas (HVDB) tiene una incidencia estimada de 33-87/1,000.000 ${ }^{1}$ correspondiendo a $20 \%$ de los sangrados digestivos ${ }^{2}$ y a un $3 \%$ de las emergencias quirúrgicas. ${ }^{1}$ Es muy importante encontrar la causa de sangrado para poder definir manejo, el cual puede ser endoscópico o quirúrgico y en algunas ocasiones, si se observa extravasación del medio de contraste en angiografía, se puede realizar embolización mesentérica, sin embargo, esta práctica sólo se realiza en menos de $1 \%$ de los casos. ${ }^{3}$ La trasfusión de hemoderivados es una de las prácticas más comunes en cuanto a intervención en HVDB. ${ }^{1}$

\section{PRESENTACIÓN DEL CASO}

Paciente de 63 años que ingresa por cuadro clínico de aproximadamente un mes de evolución de dolor abdominal generalizado, asociado a deposiciones líquidas en ocasiones deposiciones melénicas, palidez generalizada, astenia y adinamia. Como antecedentes presenta hipertensión arterial en manejo con losartán y quirúrgicos herniorrafia umbilical y osteosíntesis de miembro superior. Consumo de alcohol desde los 20 años hasta hace tres años hasta la embriaguez. Al examen físico se encuentra paciente con palidez generalizada, hemodinámicamente estable, tendencia a la hipotensión, con leve dolor a la palpación en epigastrio, sin signos de irritación peritoneal, no masas, no megalias, evidencia de melena al tacto rectal, sin evidencia de masas ni otras lesiones.

Se considera realización de endoscopía de vías digestivas altas y colonoscopía para determinar sitio de sangrado. Primera endoscopía reporta gastritis eritematosa antral, colonoscopía con mala preparación por encontrar abundantes restos hemáticos a nivel de válvula ileocecal, sin evidenciar sitio de sangrado. Se repite nuevamente endoscopía y colonoscopía sin encontrar sitio de sangrado activo, con enfermedad diverticular, restos hemáticos cerca a válvula ileocecal. Es valorado por cirugía vascular, realizan arteriografía, sin embargo, no se evidencia sitio de sangrado activo.

Se considera paciente quien presenta leve mejoría clínica, con estabilidad hemodinámica, por lo que se sugiere realización de cápsula endoscópica, sin disponibilidad de estudio en la institución, por lo que se solicita gammagrafía con leucocitos marcados que reporta hemorragia digestiva activa baja (Figura 1), originada a nivel del aspecto proximal del colon ascendente.

Vuelve a presentar melena así como requerimiento de soporte vasopresor y tiene que ser politransfundido por hemoglobina de 7.6, por lo que se decide entonces llevar a laparotomía con endoscopía intraoperatoria, sin posibilidad de enteroscopía debido a su deterioro clínico. Se realiza inicialmente colonoscopía, explorando hasta el ciego identificando válvula ileocecal y orificio apendicular, abundante sangre fresca en todo el colon de predominio en ciego, sin identificar sitio de sangrado activo, observando salida de sangre por la válvula ileocecal sin lograr ingresar al íleon distal. En colon izquierdo se observan abundantes divertículos de boca angosta sin sangrado activo. Se procede entonces a realizar enterotomía a $100 \mathrm{~cm}$ de válvula ileocecal, realizando enteroscopía hacia proximal hasta ángulo de Treitz, encontrando mucosa normal sin signos de sangrado, líquido bilioso escaso y hacia distal hasta ciego encontrando abundante contenido hemático en ciego e íleon distal (Figura 2), válvula ileocecal normal, en los últimos $60 \mathrm{~cm}$ de íleon terminal, lesiones pequeñas de aspecto angiectásico, sin encontrar sangrado activo, a nivel del ciego se encontraba sangrado a pesar de realizar lavado con abundante agua, pero no se encontraba lesión que genere el mismo, el resto de mucosa de íleon normal, marcada distensión de colon y asas delgadas, anatomía usual de colon ascendente y transverso, apéndice macroscópicamente normal. No se encuentran otras lesiones a nivel intraabdominal, se cierra enterotomía y se procede a realizar hemicolectomía derecha y resección de íleon terminal a $60 \mathrm{~cm}$ proximales de válvula ileocecal (Figura 3) con anastomosis íleo-transversa latero lateral con sutura mecánica anisoperistáltica. Posteriormente se observa ascenso de hemoglobina, no hay nueva evidencia de sangrado digestivo, tolerando vía oral y con signos claros de tránsito intestinal.

Se valora por consulta externa con reporte de patología con apéndice cecal con neoplasia mucinosa apendicular de bajo grado no infiltrante, no se observa proceso inflamatorio en la mucosa lesión tumoral, cambios vasculares importantes ni lesiones isquémicas, 11 ganglios linfáticos del tejido graso sin compromiso tumoral.

\section{DISCUSIÓN}

Una de las causas más comunes de HVDB es la enfermedad diverticular, seguido de enfermedades anorrectales como las hemorroides, fisuras y úlceras rectales, las cuales pueden verse exacerbadas en pacientes que presentan anticoagulación o algún tipo de coagulopatía; otras causas son las angiectasias, proctitis, colitis, cáncer colorrectal y pólipos. Sin embargo, hasta un $23 \%$ de los pacientes con HVDB son dados de alta sin diagnóstico. ${ }^{1}$ En esto radica la importancia de este reporte de caso, en el que se encuentra un sangrado digestivo sin causa aparente, con hallazgo patológico de tumor apendicular, lo cual no es algo frecuente.

En este caso, dada la clínica del paciente, se consideró hemorragia digestiva alta como causa de la sintomatología de ingreso, sin embargo, se realizaron dos endoscopías de vías 


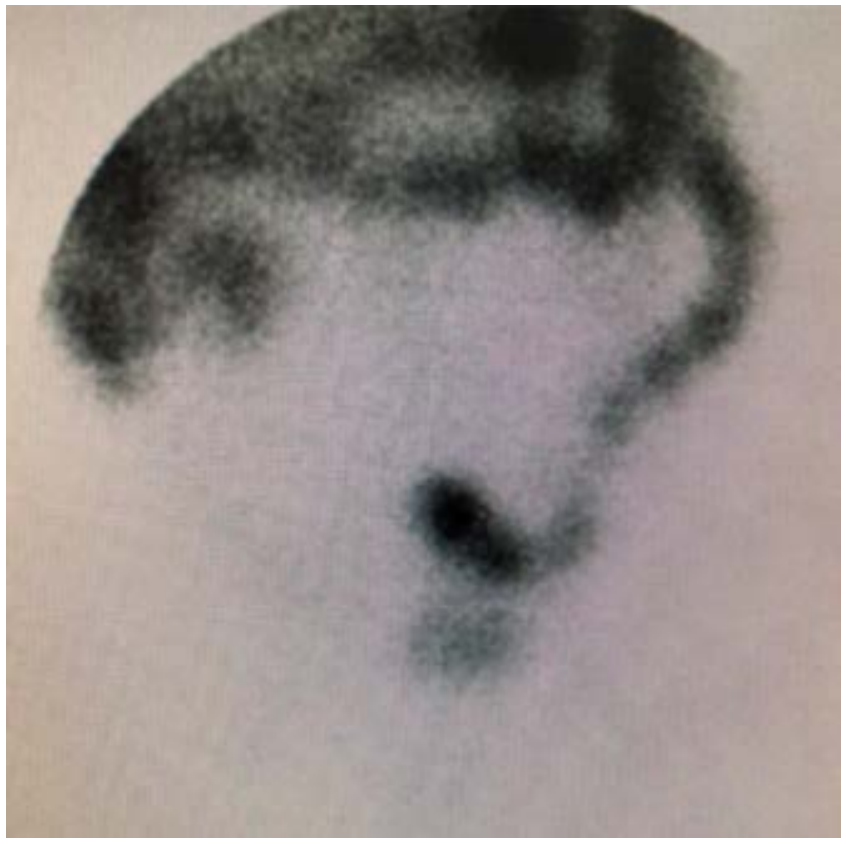

Figura 1: Gammagrafía con leucocitos marcados que reporta hemorragia digestiva activa baja.

digestivas altas sin evidencia de lesiones ni sangrado activo. Es importante tener en cuenta que aproximadamente de 11 a $15 \%$ de los pacientes en los que se sospecha sangrado digestivo bajo se encuentra finalmente un sangrado alto. ${ }^{4}$

Se pueden utilizar varios tipos de imágenes para apoyar el diagnóstico del sangrado digestivo, como la tomografía, angiografía, medicina nuclear, siendo esta última útil en caso de tener el resto negativo, incluida la colonoscopía, dando una sensibilidad de $60-93 \%,{ }^{5}$ pero con la tomografía se puede tener una mejor localización anatómica del sangrado. La cápsula endoscópica permite examinar el trayecto del intestino delgado, para encontrar lesiones que causen sangrado en este sitio. Se usa en pacientes con sangrado de origen incierto en los que, a pesar de todas las imágenes y estudios endoscópicos, aún no se encuentra la causa. Dentro de las primeras 48 horas de presentación se puede realizar un diagnóstico de hasta 87 a $91 \%$ y puede ser menor de $50 \%$ cuando se realiza después de tres días de la presentación; se debe tener en cuenta que una de las complicaciones como la retención de la cápsula ocurre hasta en $2 \%$ de los pacientes que son llevados a este estudio. ${ }^{1}$ Sin embargo, en la institución no se cuenta con éste, por lo que no fue posible realizarlo.

La angiografía con embolización también es un recurso útil cuando se cuenta con el servicio, en caso de tener un paciente hemodinámicamente estable y sirve aún más entre más rápido se realice, puesto que, dentro de los 90 minutos de una tomografía positiva para sangrado, es más factible encontrar el sangrado activo. ${ }^{6}$ En este caso, se realizó un poco tardío y no se encontró evidencia de sangrado, y no se consideró realización de enteroscopía dada la inestabilidad que presentó el paciente.

Cuando no se puede resolver el sangrado mediante técnica endoscópica o por radiología intervencionista, se tiene como última opción la laparotomía, ${ }^{3}$ excepto en casos especiales como una fístula aortoentérica, ya que se pueden realizar cirugías grandes con alta carga de morbimortalidad al no tener la localización específica del sangrado, en estos casos es menester la colaboración con el endoscopista para poder definir más fácil el sitio de sangrado.

Ahora, el tumor mucinoso del apéndice es un tumor apendicular raro, menos de $1 \%$ de los casos rara vez generan metástasis fuera de la cavidad peritoneal, ${ }^{7}$ la mayoría de pacientes tienen una presentación inespecífica, con dolor abdominal simulando apendicitis a causa de la distensión de la luz apendicular y obstrucción del orificio apendicular. ${ }^{8}$ Otros síntomas que se pueden producir son dolor abdominal crónico, pérdida de peso, anemia, infertilidad, hernia inguinal o umbilical, ${ }^{9}$ síntomas urinarios, cambios en hábito intestinal, hemorragia digestiva baja, incluso metrorragia ${ }^{10}$ o en algunas ocasiones con implantes peritoneales. ${ }^{11}$ Hasta en un $50 \%$ de los pacientes el diagnóstico se realiza como hallazgo intraoperatorio o por imágenes solicitadas por otra causa. ${ }^{10}$ Se ha descrito que presenta una mayor prevalencia en mujeres $4: 1$, aunque en otros trabajos se encuentra

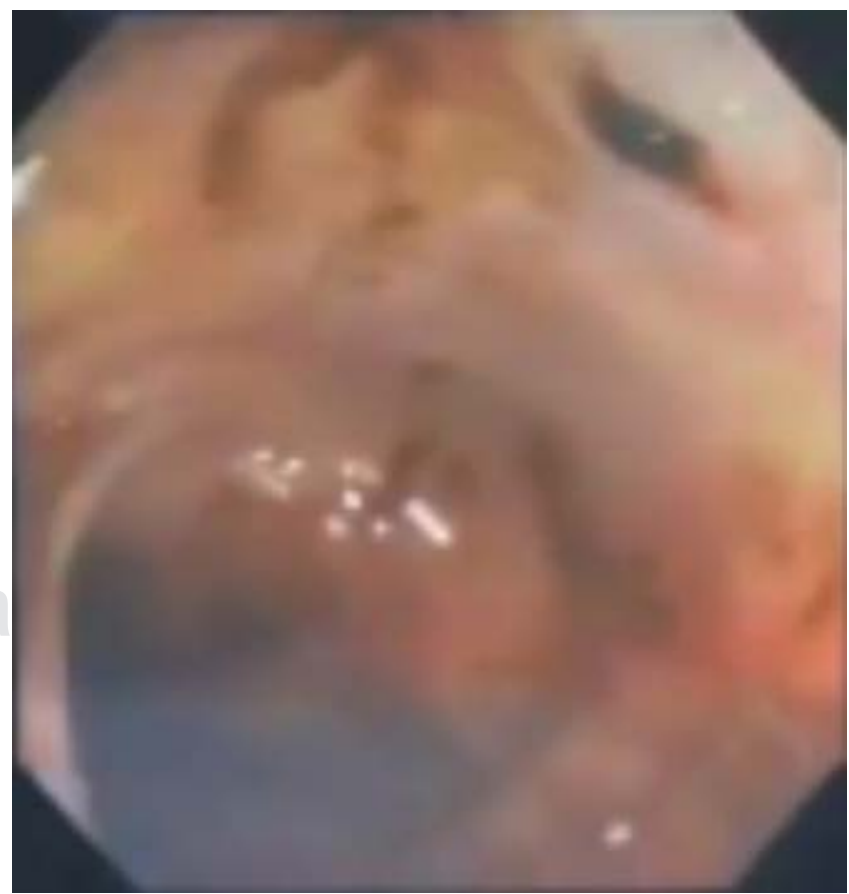

Figura 2: Contenido hemático en ciego e íleon distal. 


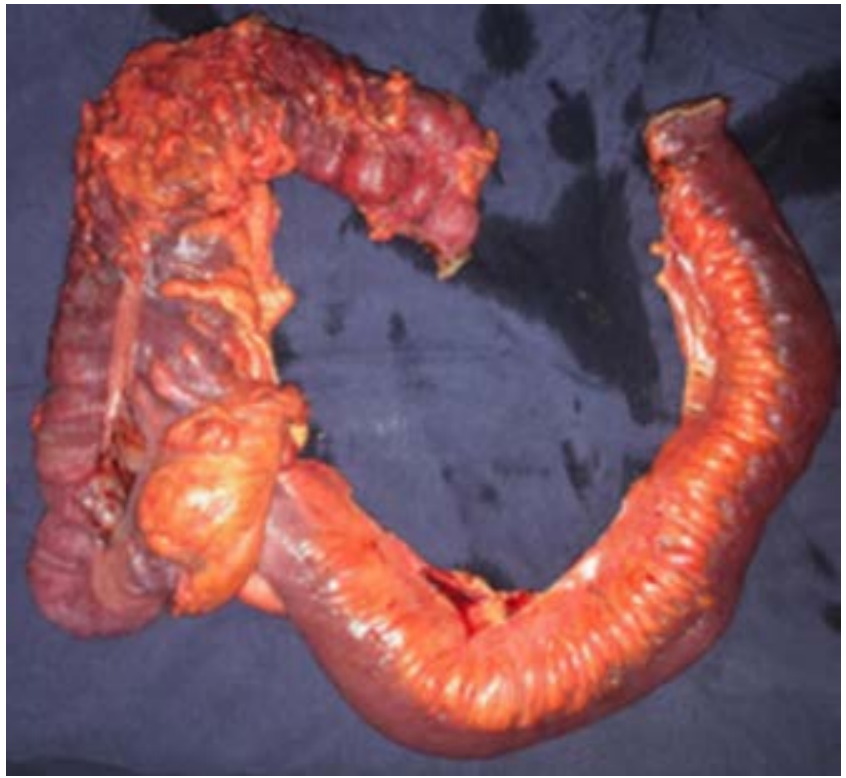

Figura 3: Hemicolectomía derecha y resección de íleon terminal a 60 $\mathrm{cm}$ proximales de válvula ileocecal.

que es más frecuente en hombres. La edad promedio de presentación es de 55 años, $75 \%$ se encuentra entre la quinta y sexta década de la vida. ${ }^{12}$

Para el tratamiento, se sugiere la apendicectomía para tumores que están localizados. Se puede definir hemicolectomía derecha para tener bordes de sección libres de tumor o si el tamaño del tumor es mayor o igual a $2 \mathrm{~cm}$, alto grado histológico, o que el tumor invada la muscular propia. ${ }^{13}$ Para disminuir el riesgo de recurrencia local, en caso de tener implantes peritoneales o el tumor perforado, se debe definir según estadio de tumor, inicio de quimioterapia o realización de citorreducción y quimioterapia intraperitoneal hipertérmica (HIPEC).

\section{CONCLUSIONES}

En este caso específico, en el que el paciente tenía un estadio temprano, la realización de la hemicolectomía derecha fue el tratamiento definitivo para el paciente, teniendo en cuenta la presentación de sangrado digestivo del tumor, con sospecha de sangrado por angiectasia en íleon terminal, lo cual fue la indicación inicial del procedimiento quirúrgico, en el que se obtiene un diagnóstico definitivo con el reporte de la patología.

\section{REFERENCIAS}

1. Oakland K Chadwick G, East JE et al. Diagnosis and management of acute lower gastrointestinal bleeding: Guidelines from the British Society of Gastroenterology. Gut. 2019; 68: 776-789. doi: 10.1136/gutjnl-2018-317807.

2. Strate LL, Gralnek IM. Management of patients with acute lower gastrointestinal bleeding. Am J Gastroenterol. 2016; 111: 459-474. doi: 10.1038/ajg.2016.41.

3. Oakland K, Guy R, Uberoi R et al. Acute lower Gl bleeding in the UK: Patient characteristics, interventions and outcomes in the first nationwide audit. Gut. 2018; 67: 654-662.

4. Laine L, Shah A. Randomized trial of urgent vs. elective colonoscopy in patients hospitalized with lower GI bleeding. Am J Gastroenterol. 2010; 105: 2636-2641.

5. O'Neill BB, Gosnell JE, Lull RJ et al. Cinematic nuclear scintigraphy reliably directs surgical intervention for patients with gastrointestinal bleeding. Arch Surg. 2000; 135: 1076 1081. discussion 81-2.

6. Koh FH, Soong J, Lieske B et al. Does the timing of an invasive mesenteric angiography following a positive CT mesenteric angiography make a difference? Int J Colorectal Dis. 2015; 30: 57-61.

7. Shaib, Assi, Shamseddine A et al. Appendiceal mucinous neoplasms: Diagnosis and management. Oncologist. 2017; 22: 1107-1116.

8. Bradley RF, Stewart JH 4th, Russell GB et al. Pseudomyxoma peritonei of appendiceal origin: A clinicopathologic analysis of 101 patients uniformly treated at a single institution, with literature review. Am J Surg Pathol. 2006; 30: 551-559.

9. Garg PK, Prasad D, Aggarwal S et al. Acute intestinal obstruction: An unusual complication of mucocele of appendix. Eur Rev Med Pharmacol Sci. 2011; 15: 99-102.

10. Avila CP, Jensen B C, Azolas MR et al. Mucocele apendicular. Reporte de un caso clínico. Cuad Cir. 2004; 18: 43-47.

11. Young RH. Pseudomyxoma peritonei and selected other aspects of the spread of appendiceal neoplasms. Semin Diagn Pathol. 2004; 21: 134-150.

12. Jiménez-Mazure C, Ramírez-Plaza CP, Carrasco C) et al. Mucocele apendicular. Actualización del tratamiento en un apatología poco conocida. Cir Andal. 2009; 20: 203-306.

13. Gonzalez-Moreno S, Sugarbaker PH. Right hemicolectomy does not confer a survival advantage in patients with mucinous carcinoma of the appendix and peritoneal seeding. Br J Surg. 2004; 91: 304-311. 